\title{
Performance Analysis of Coherent and Non-Coherent Modulation Schemes in Faded Wireless Channels using ANN-assisted MRC
}

\author{
Gargi Kar Purkayastha \\ Department of Electronics and \\ Communication Engineering \\ Girijananda Chowdhury Institute of \\ Management and Technology, \\ Guwahati-781017, Assam, India
}

\begin{abstract}
The objective of this paper is to compare the performance of coherent and non-coherent modulation schemes with the use of error correcting codes and Artificial Neural Network (ANN) assisted equalization as an aid to Maximal Ratio Combining (MRC) in order to improve bit error rate (BER) values of demodulated signals in wireless channels. The wireless channels are assumed to have both Gaussian and multipath fading characteristics. The work mainly focuses on the performance check of coherent and non-coherent modulation schemes in Rayleigh fading channel. Modulation technique used in this work are Bipolar Phase Shift Keying (BPSK), which is a coherent scheme and Differential Phase Shift Keying (DPSK), which is a non-coherent scheme in Gaussian and multipath Rayleigh fading channels. The work adopts a few error correction codes and the use of ANN block as part of a MRC set-up and is tested under SNR variation between -10 to $10 \mathrm{~dB}$ in coherent and non-coherent Gaussian and multipath fading channels. In order to demonstrate the advantages of modulation schemes that do not require phase synchronization, BER performances of BPSK are compared with the corresponding differential PSK modulation (DPSK).
\end{abstract}

\section{Keywords}

AWGN, Rayleigh, MRC, ANN, Block Codes, BPSK, DPSK

\section{INTRODUCTION}

For coherent communication systems, error performance are usually evaluated by assuming that a perfect phase reference is available in the receiver for demodulation [1][2]. In practice, this local phase reference is, however, reconstructed from a noise-corrupted version of a received signal, and thus a phase error, $\theta$, is usually results. The immediate effect of the phase error is degradation of detection performance of the coherent systems. Over the years, many researchers have investigated the error performance of binary phase shift keying (BPSK) and differential PSK (DPSK) systems over an additive white gaussian noise (AWGN) channel in the presence of noisy phase reference. This work is related to the design of an MRC scheme assisted by an Artificial Neural Network (ANN) block using some error correcting codes so as to investigate the performance of the MRC - ANN assisted equalization combination in Gaussian and multipath fading channels. The work is carried out for both coherent and noncoherent modulation shemes in Gaussian and Rayleigh fading channels with SNR variation in the range -10 to $10 \mathrm{~dB}$. The main objective is to check the performance difference in terms of the bit error rate (BER) rate of the ANN-assisted MRC for both the modulation schemes used. The rest of the paper is organised as below: Section 2 contains the basics of MRC, ANN and related considerations. Section 3 includes the details of the proposed system model. Experimental results are included in Section 4. The work is concluded by Section 5 .

\section{BACKGROUND PRINCIPLES}

The following sections provide a brief description about the constituent components as given below:

\subsection{Coherent and Non-Coherent schemes}

In many practical situations, especially in wireless communication systems, transmission channels typically are time variant due to oscillator instabilities, phase noise and motion of transmitter, receiver and / or scatterers. In digital communications, there are essentially two approaches to signal detection:

Coherent Detection: Here, the instantaneous channel state is estimated explicitly, usually based on the transmission of pilot symbols and signal detection is subsequently performed using the resulting channel estimate [3].

Noncoherent Detection: Here, the explicit estimation of the instantaneous channel state is avoided. Instead channel estimation is either performed implicitly in signal detection or even avoided entirely [3].

While the coherent approach to signal detection based on the separation of the detection problem into explicit channel estimation and signal detection is most commonly deployed in digital transmission systems, the noncoherent approach appears to be more natural, since the receiver is usually primarily interested in the transmitted information, but not in information about the current state of the channel. Furthermore, noncoherent detection schemes are more robust in rapidly varying transmission scenarios than their coherent counterparts, which rely on the accuracy of the externally obtained channel estimates [3]. They are therefore particularly apt for

1. discontinuous transmission, where coherent transmission would require a relatively large portion of pilot symbols for accurate channel estimation,

2. systems with low cost, high frequency components, where e.g. strong fluctuations of phase and frequency of local oscillators may occur, and

3. systems with time-variant interferences. 


\subsection{MRC}

It represents a theoretically optimal combiner over fading channels as a diversity scheme in a communication system. Theoretically, multiple copies of the same information signal are combined so as to maximise the instantaneous SNR at the output. Out of several diversity techniques, MRC is preferred due to the fact that it maximizes the correct reception and reduces inter-symbol interference (ISI) [5]. Figure 1 shows a MRC block.

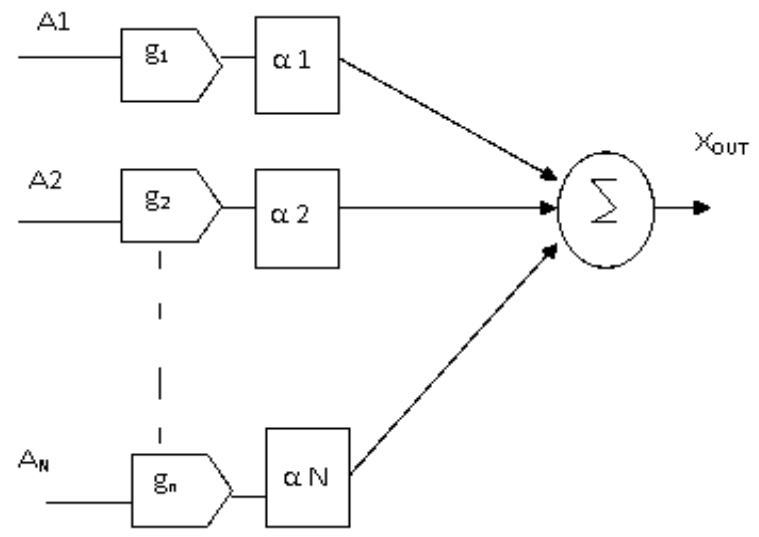

Fig. 1 MRC Block

\subsection{ANN}

An ANN is a non-parametric tool that can learn from the surrounding and use the knowledge for subsequent processing. ANN can be configured for an application as Multilayer Perceptron (MLP), which uses a feedforward topology with error back propagation principles for learning. Various training algorithms exist for MLP. The back propagation algorithm is the principle procedure for training MLPs. MLPs consist of units arranged in layers. Each layer is composed of nodes and in the fully connected network considered here each node connects to every node in subsequent layers. Each MLP is composed of a minimum of three layers consisting of an input, one or more hidden layers and an output layer [4]. Figure 2 shows a typical three layer MLP.

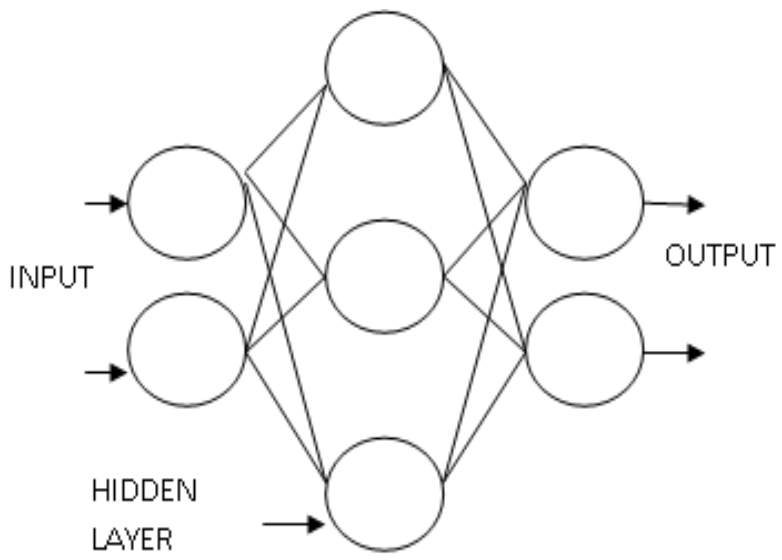

Fig. 2 Typical three layer multilayer perceptron neural network

\section{PROPOSED SYSTEM MODEL}

The experimental work carried out can be summarized by the block diagram shown in Figure 3 .

\section{DATA SOURCE}

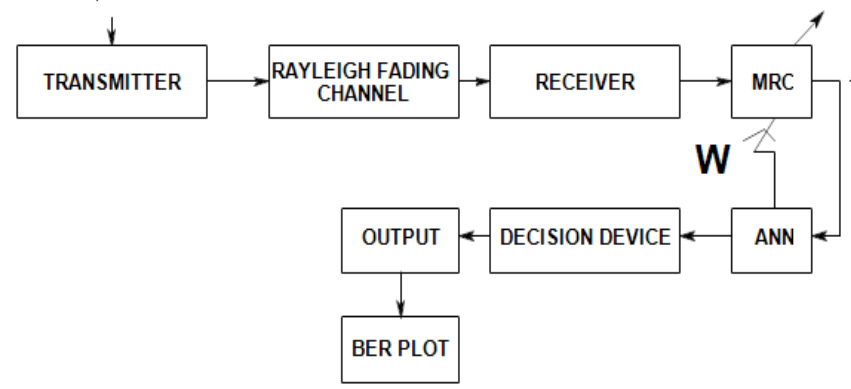

Fig. 3. Block Diagram of ANN assisted MRC

Initially uncoded data in binary form are passed through a Rayleigh faded multipath channel using both BPSK and DPSK. The Rayleigh faded channel is simulated using the parameters shown in Table 1 [8][9]. Next, coded data are modulated using both BPSK and DPSK modulation schemes and then passed through a Rayleigh fading channel. At the receiver end, multiple signals are received. After the receiver block, there is a combiner block (MRC), where the signals from all the branches are weighted according to their SNR, cophased and summed. MRC produces an output SNR equal to the sum of individual SNRs. A fixed threshold SNR is set. The updating process of the weights of the MRC system will be linked to the ANN in such a way that the process continues adaptively till the SNR crosses the fixed value. And finally the BER plot is obtained.

Table 1. Parameters used for simulating channel using Clarke-Gans model

\begin{tabular}{|l|l|l|}
\hline SI Num & Parameter & Value \\
\hline 1 & Freq., $\mathrm{f}_{\mathrm{c}}$ & $900 \mathrm{MHz}$ \\
\hline 2 & $\omega_{\mathrm{c}}$ & $2 \pi \mathrm{f}_{\mathrm{c}}$ \\
\hline 3 & Mobile Speed, $\mathrm{V}$ & $3 \mathrm{kmph}$ \\
\hline 4 & No. Of paths & 8 \\
\hline 5 & Wavelength, $\lambda$ & $3 * 10^{8} / \mathrm{f}_{\mathrm{c}}$ \\
\hline 6 & Doppler shift, $\mathrm{f}_{\mathrm{m}}$ & $\mathrm{V} / \lambda$ \\
\hline 7 & Sampling Freq, $\mathrm{f}_{\mathrm{s}}$ & $8^{*} \mathrm{f}_{\mathrm{m}}$ \\
\hline 8 & No. Of Samples, $\mathrm{N}$ & $10^{3}$ to $10^{6}$ \\
\hline 9 & Paths & 16 \\
\hline 10 & Sampling Period, $\mathrm{T}_{\mathrm{s}}$ & $1 / \mathrm{f}_{\mathrm{s}}$ \\
\hline
\end{tabular}




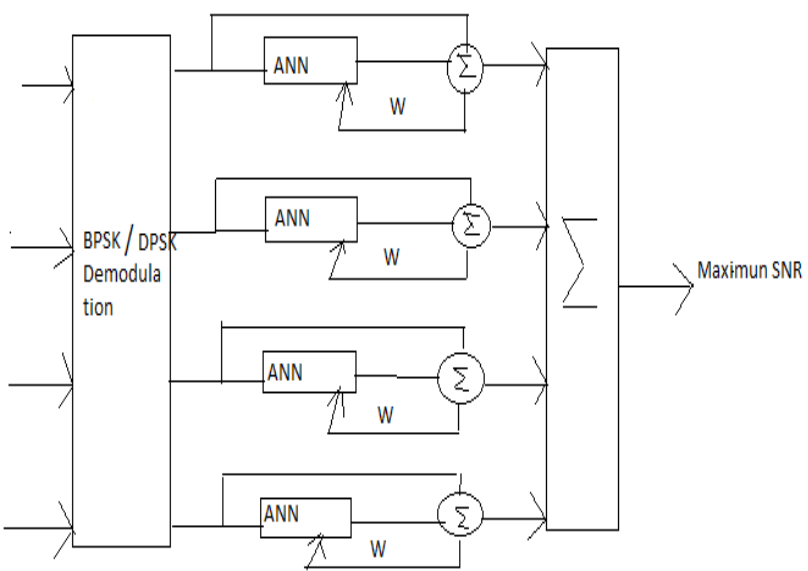

Fig. 5 Proposed model of ANN assisted MRC

\section{EXPERIMENTAL RESULTS}

The proposed system model is depicted in Figure 5. The ANN can be trained to make it robust enough to deal with multiple channel types and improve BER values. The greatest strength of an ANN is that it can tackle any estimation problem despite the presence of irregularities. The ANN learns during the training process and has the ability to recall its learning for future perceptions. The signal at the receiver is captured by several diversity branches and passed on to the demodulation block. Before demodulation and reconstruction, there is an ANN block which is combined with the MRC to make improvement in the SNR of the received signal. The ANN is trained using the Levenberg-Marquardt backpropagation algorithm and is provided a mean square error (MSE) convergence goal of $10^{-3}$. The ANN takes around 500 iterations on an average to reach this goal for about data set of size $10^{3}$. After the channels are generated the modulated signals are convolved to provide the transmission effect The detailed working of the block has already been described in Section III. . Experiments are carried out with and without the error correcting codes along with the ANN block. Results are derived from the set-up for SNR ranges between -10 and 10 $\mathrm{dB}$. Figure 6 shows the comparison plot of MRC with ANN for both the coherent (BPSK) [6] and non-coherent (DPSK) modulation schemes. Some earlier work of similar nature is reported in [5].

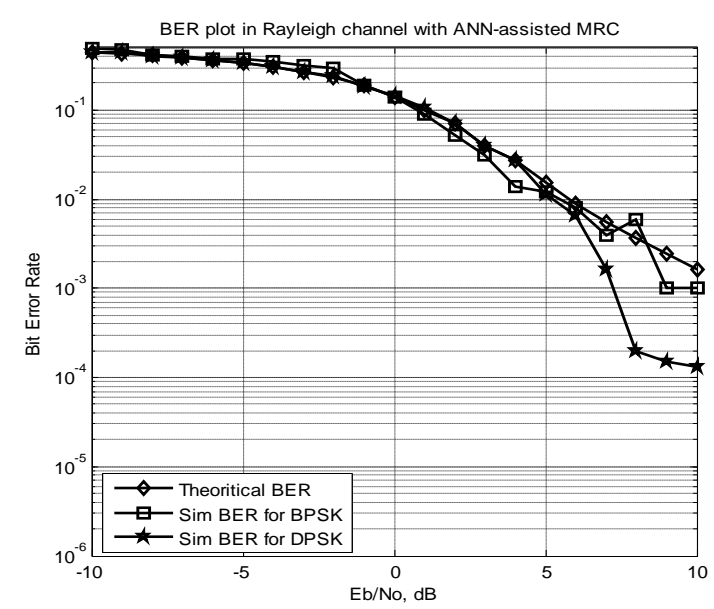

Fig. 6 BER plot generated from values obtained by using MRC technique with ANN for BPSK and DPSK schemes.
Here, it is seen that the BER values have improved when ANN-assisted equalization with DPSK modulation as compared to BPSK modulation is used. From the plot in Figure 6 , it is seen that at $5 \mathrm{~dB}$, there is an improvement in the BER values by $22.58 \%$ when BPSK modulation is used whereas the BER improved by $26.45 \%$ when DPSK modulation is used. At $7 \mathrm{~dB}$, the BER values improved by $27.35 \%$ and $70.94 \%$ when BPSK and DPSK modulations are used respectively. At $9 \mathrm{~dB}$, there is a further improvement of $58.98 \%$ and $93.84 \%$ after using BPSK and DPSK modulations respectively. So from the results it is seen that as the SNR increases, there is a significant improvement in the BER values with the use of ANN- assisted equalization for a DPSK modulated signal. The gain in percentage is given in Table 2 .

Table 2. Percentage gain in BER with use of ANN for BPSK and DPSK schemes

\begin{tabular}{|l|l|l|}
\hline SNR & BPSK & DPSK \\
\hline 5 & 22.58 & 26.45 \\
\hline 7 & 27.35 & 70.94 \\
\hline 9 & 58.98 & 93.84 \\
\hline
\end{tabular}

Figure 7 shows the comparison plot of MRC with ANN and error correcting codes for both the coherent (BPSK) [7] and non-coherent (DPSK) modulation schemes.

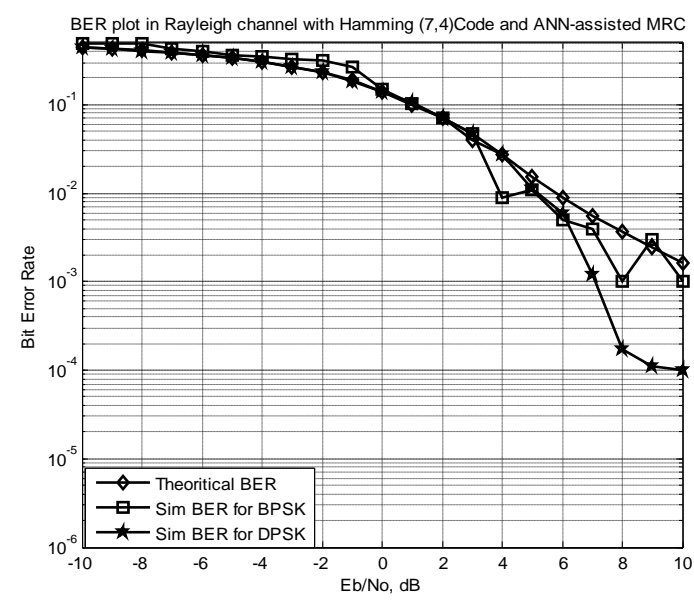

Fig. 7 BER plot generated from values obtained by using MRC technique with ANN and coding for BPSK and DPSK schemes.

Here, it is seen that there is further improvement in BER values when ANN- assisted equalisation with error correcting codes are used. From Figure 7, it is seen that at $7 \mathrm{~dB}$ the BER values improved by $27.35 \%$ with BPSK whereas the BER values further improved by $78.20 \%$ with DPSK when some error correcting codes are used with the ANN-assisted MRC. At $8 \mathrm{~dB}$, there is an improvement of $72.84 \%$ and $95.38 \%$ when BPSK and DPSK modulation schemes are used respectively. So from the results, the use of the error correcting codes with ANN block in the MRC system is justified for the non-coherent modulation scheme. The gain in percentage is given in Table 3. 
Table 3. Percentage gain in BER with use of Coding and ANN for BPSK and DPSK schemes

\begin{tabular}{|l|l|l|}
\hline SNR & BPSK & DPSK \\
\hline 7 & 27.35 & 78.20 \\
\hline 8 & 72.84 & 95.38 \\
\hline
\end{tabular}

\section{CONCLUSION}

In this work, non-coherent receivers for transmission over Rayleigh fading channels have been considered. In particular, transmission over highly time- and / or frequency-selective channels is considered here, where coherent schemes become bandwidth inefficient due to an increasing overhead in pilot symbols. It can also be said that the phase information being insignificant, has contributed towards improved performances of the non-coherent schemes. At the receiver end, after the MRC stage, when an ANN equalizer is placed and linked to adaptively update the connecting weights of the MRC system, the BER improves considerably in the non-coherent scheme as compared to the coherent ones. The improvement is noticeable despite a SNR range of around -10 to $10 \mathrm{~dB}$. The work can be further extended with the use of Self Organising Maps(SOM) instead of the MRC set-up with the same SNR variations. The work on an extended scale is applicable to mobile and wireless communication transmitting data through multipath fading channels.

\section{REFERENCES}

[1] A. J. Viterbi, Principles of Coherent Communication. New York: McGraw-Hill, 1966.

[2] W. C. Lindsey and M. K. Simon, Telecommunication Systems Engineering. Englewood Cliffs, NJ: PrenticeHall, 1973.

[3] Volker Pauli, Design and Analysis of Low-Complexity Noncoherent Detection Schemes, May 7, 2007.

[4] S.Haykins:NeuralNetworks A Comprehensive Foundation, Pearson Education, $2^{\text {nd }}$ ed., New Delhi, 2003.

[5] A. Choudhury and K.K.Sarma: Adaptive Equalization assisted MRC in WirelessChannels, International Conference on Information, Signal and Communication (ICISC-2011), New VallabhVidyanagar, India, Feb.,2011.

[6] G. K. Purkayastha and K. K. Sarma: “ Maximal Ratio Combining using ANN- Assisted Equalisation in Wireless Channels", IEEE $2^{\text {nd }}$ National Conference on Computational Intelligence and Signal Processing (CISP2012), pp. 1-4, Guwahati, India, March, 2012.

[7] G. K. Purkayastha and K. K. Sarma: “ Maximal Ratio Combining using Coding and ANN- Assisted Equalisation in Wireless Channels", 57 $7^{\text {th }}$ Annual Technical Session Assam Science Society, pp. 1-5, Guwahati, India, March, 2012.

[8] T. S. Rappaport: "Wireless Communications: Principles and Practice, Second Edition," Pearson Education, 7th Indian Reprint, 2004.

[9] J. G. Proakis: "Digital Communications, 4th ed.," McGraw Hill Publication,2001. 